\title{
Symmetric Symbolic Safety-Analysis of Concurrent Software with Pointer Data Structures
}

\author{
Farn Wang $^{1 \star}$ and Karsten Schmidt ${ }^{2 \star \star}$ \\ 1 Inst. of Info. Science, Academia Sinica, Taipei, Taiwan, R.O.C. \\ farn@iis.sinica.edu.tw \\ 2 Dept. of Computer Science, Carnegie-Mellon University \\ Pittsburgh, PA, 15213, USA, kschmidt@cs.cmu.edu
}

\begin{abstract}
We formally define the model of software with pointer data structures. We developed symbolic algorithms for the manipulation of conditions and assignments with indirect operands for verification with BDD-like data-structures. We rely on two techniques, including inactive variable elimination and process-symmetry reduction in the data-structure configuration, to contain the time and memory complexity. We use binary permutation for efficiency but also identify the possibility of anomaly of image false reachability. We implemented the techniques in tool red and compare performance with Mur $\phi$ and SMC against several other benchmarks.
\end{abstract}

Keywords: Symmetry, symbolic model-checking, pointers, datastructures

\section{Introduction}

Verification of networks with special topologies like rings and buses has been widely studied. In real-world software, arbitrary and dynamic network configuration is, however, often constructed using pointers. An action like " $x_{1} \rightarrow$ $x_{2} \rightarrow \ldots \rightarrow x_{n}:=\ldots ; "$ can stretch through a network and change the local memory of a peer process in the network. Such indirect references are not only very common in practice, but also extremely important in both hardware and software engineering. For example, most CPUs now support hardware indirect addressing to facilitate virtual memory management. This important hardware indirect referencing mechanism is transparent to softwares and runs silently. For another example, dynamic data-structures like linear lists, trees, and graphs are constructed with pointers and used intensively in most nontrivial softwares. In

\footnotetext{
* The work is partially supported by NSC, Taiwan, ROC under grants NSC 90-2213E-001-006, NSC 90-2213-E-001-035, and the by the Broadband network protocol verification project of Institute of Applied Science \& Engineering Research, Academia Sinica, 2001.

** supported by DARPA/ITOP within the MoBIES project
}

D.A. Peled and M.Y. Vardi (Eds.): FORTE 2002, LNCS 2529, pp. 50-64 2002.

(C) Springer-Verlag Berlin Heidelberg 2002 


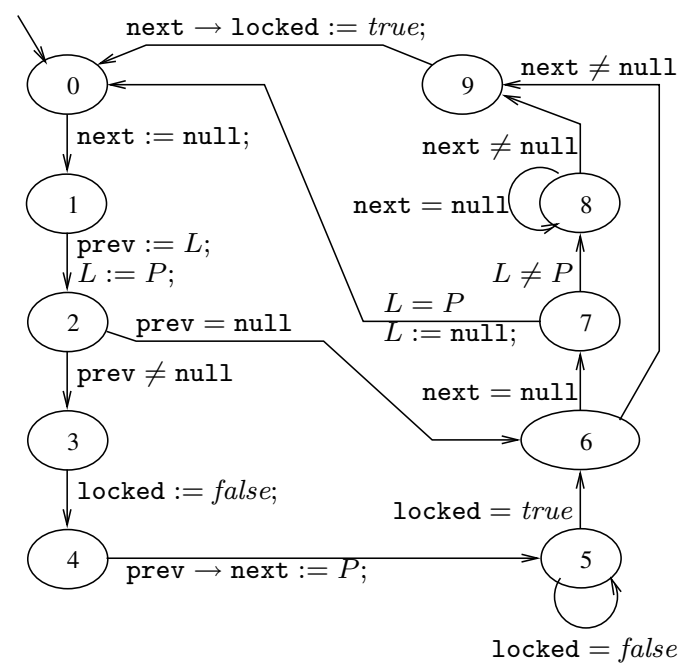

Fig. 1. MCS locking algorithm

example 1, we have a locking algorithm 12 which uses pointers to maintain a queue for critical section mutual exclusion.

Example 1: MCS (Mellor-Crummy \& Scott's) locking algorithm. The algorithm 12 is an example protocol in which a global waiting queue of processes is explicitly used to insure mutual exclusive access to the critical section in a concurrent system. In figure 1. The MCS locking algorithm for a process is drawn as a finite-state automaton. The critical section is from modes 6 to 9 . The queue is constructed with one global pointer L (to the tail of the queue), and two local pointers of each process: next and prev (respectively to the successor and predecessor processes of the local process in the queue). $P$ is a special symbol for the data structure address of the running process. Each process also has a local Boolean variable locked which is set to false when the process is permitted to the critical section by its predecessor in the queue. We want to guarantee that at any moment, at most one process is in its critical section.

Due to their dynamic nature, software with pointer data-structures has been known to be extremely difficult to maintain and to debug. For example, any experienced software engineers will agree that bugs caused by dirty pointers to freed data-structures are extremely difficult to detect and remove. Such bugs, whose effect usually does not emerge until long after a data-structure is corrupted through a dirty pointer, is very difficult to trace backward.

The technique of symbolic model-checking manipulates logic predicates describing state-spaces. Since the technique can usually handle large sets of states in an abstract and concise way, it provides opportunity for higher efficiency in verification. In the last decades, BDD (Binary-Decision Diagram) [25] has 
emerged as one of the prime industry technology in symbolic manipulation. In this paper, we have the following accomplishments.

- We define a formal model for concurrent software with pointer data structures for rigorous research on solution for the problem. Especially note that the framework is defined in such a way to explicitly allow all processes to share the same automaton template but at the same time allow them to use their local variables. This is extremely important in identifying processsymmetry in a convincing way. Most other model-checkers 3.41016] accept that each process be described with its own automaton and usually create difficulty in efficiently identifying symmetric behaviors among the process automata. (Note that asymmetric systems can also be modeled in our framework with processes running mutually disjoint parts of the program.)

- We present algorithms for the symbolic manipulation of conditions and assignments with indirect operands for verification with BDD-like datastructures. Algorithms for both forward analysis and backward analysis have been developed and implemented with tuning for verification performance. Special care is taken to allow for recurrence assignments, like $y \rightarrow x:=3 y \rightarrow x+z$; where the left-hand-side may also occur in the right-hand-side.

- We adapt two reduction techniques for model checking such systems.

- Reduction by inactive variables eliminations, which helps the construction of concise state-space representation through the elimination of variable valuations that do not affect the system behaviors 4,101415. 16. Due to the implicit reading of pointer values in the indirection of operand references, the adaptation is not so trivial.

- Reduction by process-symmetry. The idea of symmetry reduction 6816, 13.14 is to keep only one state if two states turn out to be symmetric. We shall follow the approach of process-symmetry in 814 16] since process represents a typical basic unit for behavioral equivalence in symmetry. For efficiency, we use binary permutation of process identifiers to transform data-structures to their isomorphic ones with process re-indexing. How to design a symbolic predicate to detect the necessary condition for permutation is also discussed.

- We also implemented our modelling and verification techniques for pointer data-structure systems in our model-checker red version 3.1, which is available freely at

http://www.iis.sinica.edu.tw/ farn/red/

for timed automata. The implementation not only support pointer datastructures but also support complex arithmetics on process identifiers.

- We carried out experiments on several benchmarks to show the usefulness of our techniques and compare performance with SMC [9] and Mur $\phi$ [711].

In section 2, we shall define the formal framework of this research. In section [3] we shall present the algorithmic framework to integrate safety-analysis software with various reduction techniques. In section 4, we shall present the algorithm for the manipulation of symbolic predicates and symbolic assignment 
statements with BDD-like data-structures. In section 5 , we shall discuss our reduction techniques. In section 6, we discuss our implememntations and experiments. Conclusion is omitted due to page-limit.

\section{Concurrent Algorithms and the Safety Analysis Problem}

We consider concurrent algorithms with local data structures attached to each process for convenience of presentation and discussion. The address of a data structure can be viewed as the identity of the corresponding process. We shall have the convention that if $p$ is the address of a process's data-structure, then the process is also named $p$. But our model and techniques can be easily adapted for the modelling and verification of systems with data-structure addresses not bound to process identifiers.

Two types of variables can be declared. The first is the type of discrete variables with predeclared finite integer value ranges. For each declared variable $x$, $\mathrm{lb}(x)$ and $\mathrm{ub}(x)$ denote its declared lowerbound and upperbound respectively. Such variables can be used in formulae and assignments with arithmetic expressions and indirect operands. For convenience, we can also assign symbolic macro names to integer values. Traditionally, false is interpreted as 0 while true as 1 .

The second is the type of pointers (address variables) to processes (datastructures). The value ranges of pointers are from zero (or NULL) to the number of processes. As in example 1, $L$ is used as a pointer to the tail of a queue. We also support arbitrary address arithmetics. A special pointer value constant symbol is NULL, which in C's tradition is equal to zero. Or in the same notations as of discrete variables, $\mathrm{lb}(x)=$ NULL and $\mathrm{ub}(x)$ is the number of processes for all declared pointers $x$.

Variables can be declared as global variables which all processes can access, or local variables of a process which only the declaring process can directly access. A name can be used to represent the respective local variables of different processes. For instance, in example 1 different processes access different variables which are all locally called locked.

In the following, we shall first formally define the syntax and semantics of our systems, and then define the safety analysis problem.

\subsection{Syntax of Algorithm Descriptions}

Conceptually, a concurrent algorithm $S$ is a tuple $\left(G^{d}, G^{p}, L^{d}, L^{p}, A(P)\right)$ where $G^{d}$ and $L^{d}$ are respectively the sets of global and local discrete variables, $G^{p}$ and $L^{p}$ are respectively the sets of global and local pointers, and $A(P)$ is the process program template, with process identifier symbol $P$.

Given a set $X^{d}$ of global and local discrete variables and a set $X^{p}$ of global and local pointers, a local state predicate $\eta$ of $X^{d}$ and $X^{p}$ can be used to describe the triggering condition of state transitions and has the following syntax. 


$$
\begin{aligned}
& \eta::=\epsilon_{1} \sim \epsilon_{2}|\neg \eta| \eta_{1} \vee \eta_{2} \\
& \epsilon::=c|\operatorname{NULL}| P|x| y \rightarrow \epsilon|x[p]| y[p] \rightarrow \epsilon \mid \epsilon_{1} \oplus \epsilon_{2}
\end{aligned}
$$

where $\sim \in\{\leq,<,=, \neq,>, \geq\}, c \in \mathcal{N}-\{0\}, x \in X^{d} \cup X^{p}, y \in X^{p}$, and $\oplus \in$ $\{+,-, *, /\}$. Parenthesis can be used for disambiguation. Traditional shorthands are $\epsilon_{1} \neq \epsilon_{2} \equiv \neg\left(\epsilon_{1}=\epsilon_{2}\right), \eta_{1} \wedge \eta_{2} \equiv \neg\left(\left(\neg \eta_{1}\right) \vee\left(\neg \eta_{2}\right)\right)$, and $\eta_{1} \Rightarrow \eta_{2} \equiv\left(\neg \eta_{1}\right) \vee \eta_{2}$, Thus a process may operate on conditions of the global and local variables, and also on the local variables of peer processes pointed to by pointers. We let $B\left(X^{d}, X^{p}\right)$ be the set of all local state predicates constructed on the discrete variable set $X^{d}$ and the pointer set $X^{p}$.

In our concurrent algorithms, once the triggering condition is satisfied by global variables and the local variables of a process, the process may execute a finite sequence of actions with the following syntax: " $y_{1} \rightarrow y_{2} \rightarrow \ldots \rightarrow y_{n} \rightarrow$ $x:=\epsilon ; "$ where $n \geq 0$. Conveniently, let $T\left(X^{d}, X^{p}\right)$ be the set of all finite sequences of actions constructed of discrete variable set $X^{d}$ and pointer set $X^{p}$.

Given a concurrent algorithm $S=\left(G^{d}, G^{p}, L^{d}, L^{p}, A(P)\right), A(P)$ is the program template, with identifier symbol $P$, for all processes. Program template $A(P)$ has a syntax similar to that of finite-state automata. $A(P)$ is conceptually a tuple $\left(Q, q_{0}, E, \tau, \pi\right)$ with the following restrictions:

- $Q$ is a finite set of operation modes.

- $q_{0} \in Q$ is the initial operation mode.

- $E \subseteq Q \times Q$ is the set of transitions between operation modes.

- $\tau: E \mapsto B\left(G^{d} \cup L^{d}, G^{p} \cup L^{p}\right)$ is a mapping that defines the triggering condition of each transition.

- $\pi$ : $E \mapsto T\left(G^{d} \cup L^{d}, G^{p} \cup L^{p}\right)$ is a mapping that defines the action sequence performed upon occurrence of a transition. We assume that transitions are atomic actions.

We require that there is a variable mode $\in L^{d}$ that records the current operation mode of the corresponding process. However, when drawing $A(P)$ as an automaton like in figure 1, we omit the description of mode values in the triggering conditions and action sequences for simplicity and clarity.

\subsection{Computation of Systems}

Given a system of $\mathcal{M}$ processes, we assume the processes are indexed with integer from 1 to $\mathcal{M}$. Given a concurrent algorithm $S, S^{\mathcal{M}}$ denotes the implementation of $S$ by exactly processes one through $\mathcal{M}$. A state $\nu$ of $S^{\mathcal{M}}$ is a mapping from

$$
\{\text { NULL }, 1, \ldots, \mathcal{M}\} \times\left(\mathcal{N} \cup G^{d} \cup G^{p} \cup\{\perp, \text { NULL }, P\} \cup L^{d} \cup L^{p}\right)
$$

such that

- $\nu($ NULL,$x)=\perp$ (memory fault) for all $x \in \mathcal{N}$ and all variable $x$.

- for all $1 \leq p \leq \mathcal{M}, \nu(p, \perp)=\perp ; \nu(p, P)=p ; \nu(p, c)=c$ if $c \in \mathcal{N}$; and $\circ$ for all $x \in G^{d}, \nu(p, x) \in[\operatorname{lb}(x), \mathrm{ub}(x)]$ is the value of global discrete variable $x$ at state $\nu$;

○ for all $x \in G^{p}, \nu(p, x) \in\{$ NULL $\} \cup\{1, \ldots, \mathcal{M}\}$ is the value of global pointer $x$ at state $\nu$; 
○ for all $x \in L^{d}, \nu(p, x) \in[\mathrm{lb}(x), \mathrm{ub}(x)]$ is the value of local discrete variable $x$ of process $p$ at state $\nu$; and

$\circ$ for all $x \in L^{p}, \nu(p, x) \in\{\operatorname{NULL}\} \cup\{1, \ldots, \mathcal{M}\}$ is the value of local pointer $x$ of process $p$ at state $\nu$.

Given a global state $\nu$, a process $1 \leq p \leq \mathcal{M}$, and a process predicate $\eta \in$ $B\left(G^{d} \cup L^{d}, G^{p} \cup L^{p}\right)$, we define the mapping of $p$ satisfies $\eta$ at $\nu$, written $\nu(p, \eta)$, to $\{$ true, false,$\perp\}$ in the following inductive way.

- $\nu(p, y \rightarrow \epsilon)=\nu(\nu(p, y), \epsilon)$ if $p \neq$ NULL.

- $\nu(p, y[c] \rightarrow \epsilon)=\nu(c, y \rightarrow \epsilon)$ if $1 \leq c \leq \mathcal{M}$; otherwise, $\nu(p, y[c] \rightarrow \epsilon)=\perp$.

- $\nu\left(p, \epsilon_{1} / \epsilon_{2}\right)=\perp$ if $\oplus=' /, \wedge \nu\left(p, \epsilon_{2}\right)=0$.

- $\nu\left(p, \epsilon_{1} \oplus \epsilon_{2}\right)=\nu\left(p, \epsilon_{1}\right) \oplus \nu\left(p, \epsilon_{2}\right)$ if either $\oplus \in\{+,-, *\}$ or $\oplus={ }^{\prime} /{ }^{\prime} \wedge \nu\left(p, \epsilon_{2}\right) \neq$ 0 . Integer-division is assumed, that is $x / y$ is defined as $\frac{x * y}{|x * y|}\lfloor|x / y|\rfloor$, where $\frac{x * y}{|x * y|}$ is the sign of $x / y$.

- $\nu\left(p, \epsilon_{1} \sim \epsilon_{2}\right)=\nu\left(p, \epsilon_{1}\right) \sim \nu\left(p, \epsilon_{2}\right)$

- " $\perp \sim \epsilon$ " equals to $\perp$ and " $\epsilon \sim \perp$ " equals to $\perp$.

- The negation of the satisfaction mapping is defined as follows.

$$
\begin{array}{c||c|c|c}
\nu(p, \eta) & \text { false } \perp \text { true } \\
\hline \nu(p, \neg \eta) & \text { true } \perp \text { false }
\end{array}
$$

- The disjunction of the satisfaction mapping is defined as follows.

\begin{tabular}{c||c||c|c}
$\nu\left(p, \eta_{1} \vee \eta_{2}\right)$ & false & $\perp$ & true \\
\hline \hline false & false & $\perp$ & true \\
\hline$\perp$ & $\perp$ & $\perp$ & $\perp$ \\
\hline true & true & $\perp$ & true
\end{tabular}

Given an action $\alpha$ of $S$, the new global state obtained by applying $y_{1} \rightarrow \ldots \rightarrow y_{n} \rightarrow x:=\epsilon ;$, with $n \geq 0$, to $p$ at $\nu$, written next_state $\left(p, \nu, y_{1} \rightarrow \ldots \rightarrow y_{n} \rightarrow x:=\epsilon ;\right)$, is defined as follows:

- When $\nu\left(p, y_{1} \rightarrow \ldots \rightarrow y_{n} \rightarrow x\right) \neq \perp \quad$ and $\nu(p, \epsilon) \quad \neq \perp$, next_state $\left(\nu, p, y_{1} \rightarrow \ldots \rightarrow y_{n} \rightarrow x:=\epsilon ;\right)$ is identical to $\nu$ except that next_state $\left(\nu, p, y_{1} \rightarrow \ldots \rightarrow y_{n} \rightarrow x:=\epsilon ;\right)\left(\nu\left(p, y_{1} \rightarrow \ldots \rightarrow y_{n}\right), x\right)=\nu(p, \epsilon)$.

- When either $\nu\left(p, y_{1} \rightarrow \ldots \rightarrow y_{n} \rightarrow x\right)=\perp$ or $\nu(p, \epsilon)=\perp$, next_state $\left(\nu, p, y_{1} \rightarrow \ldots \rightarrow y_{n} \rightarrow x:=\epsilon ;\right)$ is undefined.

Note that the semantics is defined to allow for recurrence of a variable in both the left-hand-side and right-hand-side of an assignment. Given an action sequence $\alpha_{1} \ldots \alpha_{n} \in T\left(G^{d} \cup L^{d}, G^{p} \cup L^{p}\right)$, we let

$$
\operatorname{next} \_\operatorname{state}\left(\nu, p, \alpha_{1} \alpha_{2} \ldots \alpha_{n}\right)=\text { next_state }\left(\text { next_state }\left(p, \nu, \alpha_{1}\right), p, \alpha_{2} \ldots \alpha_{n}\right) \text {. }
$$

The initial state $\nu_{0}$ of an implementation $S^{\mathcal{M}}$ must satisfy $\bigwedge_{1 \leq p \leq \mathcal{M}} \nu_{0}(p$, mode $)=0$. We assume that the system runs with interleaving semantics in the granularity of transitions, that is at any moment, at most one process can execute a transition. Execution of a transition is atomic.

A computation of an implementation $S^{\mathcal{M}}$ is a (finite or infinite) sequence $\rho=\nu_{0} \nu_{1} \ldots \nu_{k} \ldots \ldots$ of states such that for all $k \geq 0$,

- $\nu_{0}$ is the initial state of $S^{\mathcal{M}}$; and 
- for each $\nu_{k}$ with $k>0$, either $\nu_{k}=\nu_{k-1}$ or there is a $p \in\{1, \ldots, \mathcal{M}\}$ and a transition from $q$ to $q^{\prime}$ such that $\nu_{k-1}\left(p, \tau\left(q, q^{\prime}\right)\right)=$ true and next_state $\left(\nu_{k-1}, p, \pi\left(q, q^{\prime}\right)\right)=\nu_{k}$ is defined.

\subsection{Safety Analysis Problem}

To write a specification for the interaction among processes in a concurrent system, we need to define global predicates with the following syntax.

$$
\begin{aligned}
& \phi::=\psi_{1} \sim \psi_{2}|\neg \phi| \phi_{1} \vee \phi_{2} \\
& \psi::=c|\operatorname{NULL}| y|x[p]| z \rightarrow \epsilon|w[p] \rightarrow \epsilon| \psi_{1} \oplus \psi_{2}
\end{aligned}
$$

where $c \in \mathcal{N}, y \in G^{d} \cup G^{p}, x \in L^{d} \cup L^{p}, z \in G^{p}, w \in L^{p}$, and $1 \leq p \leq \mathcal{M}$.

Given a state $\nu$ and a global predicate $\phi$, we define the valuation of $\nu$ on $\phi$, written $\nu(\phi)$, in the following inductive way.

- $\nu\left(\psi_{1} \sim \psi_{2}\right)=\nu\left(\psi_{1}\right) \sim \nu\left(\psi_{2}\right) \in\{$ true, false $\}$

- $\nu(x[p])=\nu(p, x)$

- $\nu(\neg \phi)=\neg \nu(\phi)$.

- $\nu\left(\phi_{1} \vee \phi_{2}\right)=\nu\left(\phi_{1}\right) \vee \nu\left(\phi_{2}\right)$.

The rest is the same as the corresponding rules for local state predicates.

A computation $\rho=\nu_{0} \nu_{1} \ldots \nu_{k} \ldots \ldots$ of $S^{\mathcal{M}}$ violates safety property $\phi$ iff there is a $k \geq 0$ such that either $\nu_{k}$ is undefined or $\nu_{k}(p, \phi) \neq$ true for some $1 \leq p \leq \mathcal{M}$. The safety analysis problem instance $\operatorname{SAP}(S, \mathcal{M}, \phi)$ is to determine if for all computations $\rho$ of $S^{\mathcal{M}}$ starting from some initial states, $\rho$ does not violate safety property $\phi$.

Example 2: Consider the MCS locking algorithm in example 1. The critical section consists of modes 6 through 9 . Thus the safety analysis problem for mutual exclusive access to the critical sections of two processes can be formulated as $\operatorname{SAP}(S, 2, \neg(6 \leq \operatorname{mode}[1] \leq 9 \wedge 6 \leq \operatorname{mode}[2] \leq 9))$.

\section{Framework for Safety Analysis and Reductions}

The goal of the framework is to explore and construct a representation of the reachable state-space and analyze if the automaton ever violates safety property. Our general algorithmic framework for symbolic safety analysis is shown as follows.

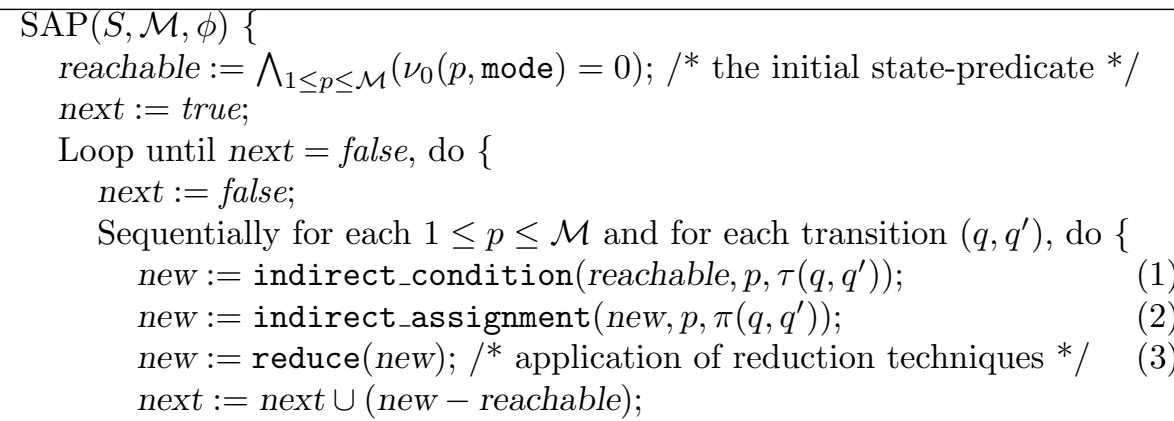




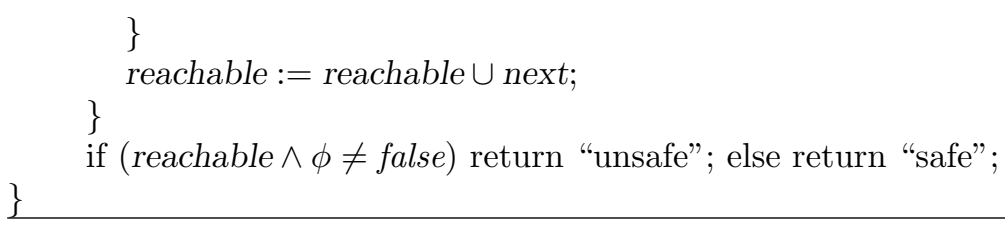

The procedure iterates through the outer loop until reachable becomes a fixpoint. At line (1), indirect_condition $(D, p, \eta)$ returns a global predicate in BDD representing the subspace of $D$ in which $\eta$ is true of process $p$. At line (2), indirect_assignment $\left(D, p, \pi\left(q, q^{\prime}\right)\right)$ calculates a global predicate in BDD representing the result after applying action sequence $\pi\left(q, q^{\prime}\right)$ to states in subspace represented by $D$. Symbolic implementations of procedure indirect_condition () and indirect_assignment () will be discussed in section 4. At line (3), reduce() is about application of various reduction techniques to control the complexity of reachable state-space representations.

At the first glance, model checking technology looks straightforward. The real challenge comes from the fact that in practice, the representation sizes of reachable state-spaces of any reasonably interesting software implementations are usually tremendous. In sections 5 , we shall present two techniques to reduce the complexity of state space representations.

\section{Manipulation of Predicates with Indirections}

In our presentation of symbolica algorithms with BDD, we shall conveniently write Boolean combinations of BDDs, like $D_{1} \vee D_{2}$, with the assumption that Boolean operations on BDDs are already defined. Details of such BDD operations can be found in 215.

\subsection{Symbolic Evaluation of Conditions with Indirect Operands}

In a pointer data-structure system, users may write a predicate with operands of aribitrary indirections. For example, we may have a pointer data-structure system with the following declarations.

global pointer L;

local pointer parent, leftchild, rightchild;

local discrete count: 0..5;

All these variables are to be encoded by finite number of bits in BDD-like datastructure. This is possible because their value ranges are finite. Specifically, $\mathrm{lb}($ count $)=0$, and $\mathrm{ub}($ count $)=5$.

When we are given a state-space representation $D$ in BDD-like datastructure, how can we compute the maximal subspace representation $D^{\prime}$, of $D$, in which a complicate condition $\eta$ with indirections like

parent $[1] \rightarrow$ count $-2 *$ leftchild $[2] \rightarrow$ rightchild $\rightarrow$ count $<L \rightarrow$ count 
is true. The condition says that difference of the count of parent of the 1st process (parent $[1] \rightarrow$ count) and twice the count of the right child of the left child of the 2 nd process $(2 *$ leftchild $[2] \rightarrow$ rightchild $\rightarrow$ count $)$ is less than the count of process $\mathrm{L}$ ( $L \rightarrow$ count). Since there is no restriction on lengths of indirections, we need a flexible algorithm to construct such $D^{\prime}$. Our algorithm is simplified for presentation and explanation as the following function indirect_condition(), which in turns invokes functions indirect_ref(), indirect_arith(), and indirect_effect().

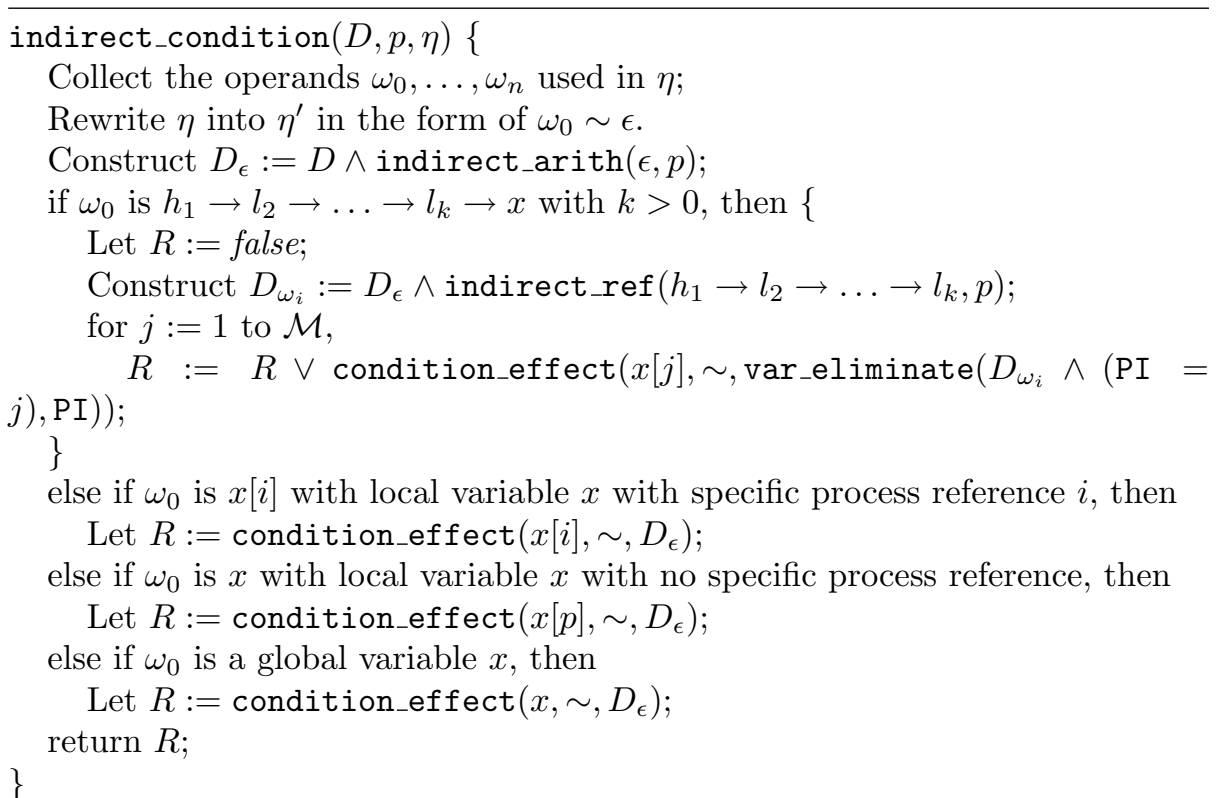

Procedure var_eliminate $(D, x)$ filters $x$ out of $D$. For a local discrete variable $x$, var_eliminate $(D, x)=\left.\bigvee_{v \in[l b(x), u b(x)]} D\right|_{x=v}$ where $\left.D\right|_{x=v}$ is the new local state predicate obtained by instantiating $x$ to $v$. For a local pointer $x$, var_eliminate $(D, x)=\left.\bigvee_{v \in\{\text { NULL }, 1, \ldots, \mathcal{M}\}} D\right|_{x=v}$.

The presentation is simplified in that when it invokes indirect_arith(), we assume that we don't have to worry about problems like divide-by-zero and imprecision caused by integer division. In our real implementation, the algorithm is more involved and iteratively solves the linear inequality constraints with respect to operand $\omega_{0}$. In the iterations to solve the inequality constraints, such problems are properly taken care of with case-analysis. Due to page-limit, we only use the simplified presentations of algorithms in the following. The algorithm uses two auxiliary variables, VALUE and PI. VALUE is used to hold the value of ann arithmetic expression. PI is used to hold the destination process identifier of an indirection of arbitrary length.

Function indirect_ref $\left(h_{i} \rightarrow l_{i+1} \rightarrow \ldots \rightarrow l_{k}, p\right)$ constructs the necessary condition at a state $\nu$ when $\nu\left(h_{i} \rightarrow l_{i+1} \rightarrow \ldots \rightarrow l_{k}, p\right)$ is identical to the process identifier recorded in variable PI. 


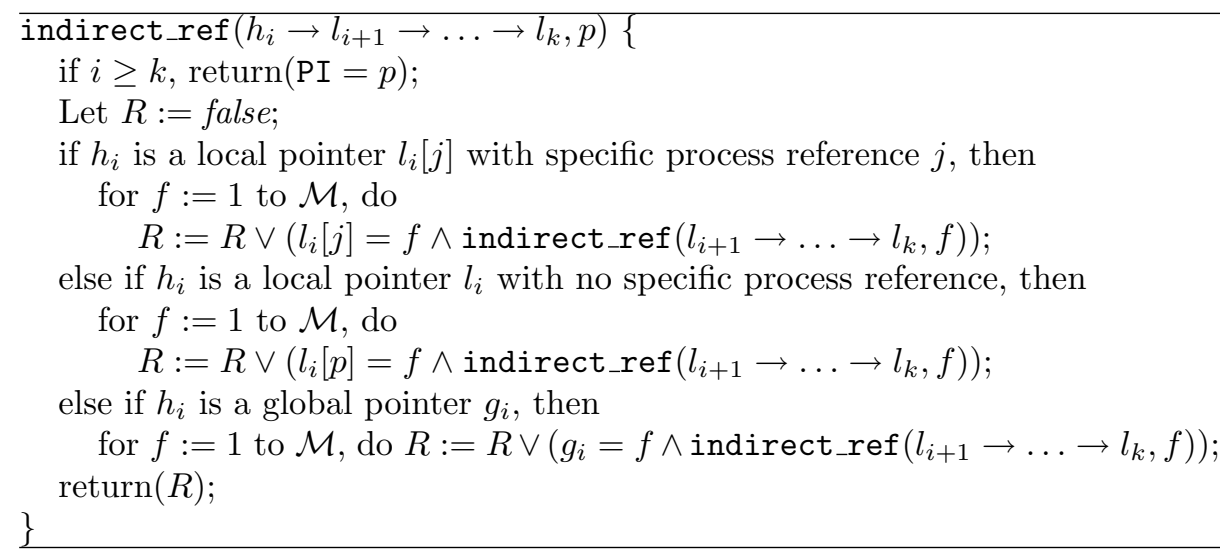

Due to page-limit, we briefly describe the functions of the other procedures. Function indirect_arith $(\epsilon, p)$ uses the auxiliary variable VALUE to symbolically record the value of expression $\epsilon$ at process $p$. It returns the state-predicate with VALUE to the value of expression $\epsilon$ for process $p$ at every state.

To evaluate an expression like $\epsilon_{1} \oplus \epsilon_{2}$, the values recorded in the VALUE variable respectively in the symbolic predicates of $\epsilon_{1}$ and $\epsilon_{2}$ are used as in line (1) in procedure indirect_arith() are pairwisely compared and corresponding state-predicate conjuncted.

\subsection{Symbolic Assignments with Indirect Operands}

Given a state-space predicate $D$ and an assignment statement like $\omega_{0}:=\epsilon$; the symbolic postcondition by process $p$ in traditional wisdom is

$$
\text { indirect_condition(var_eliminate } \left.\left(D, \omega_{0}\right), p, \omega_{0}=\epsilon ;\right)
$$

But this fails in two ways. First, there can be indirections in $\omega_{0}$. Second, the destination of $\omega_{0}$ can occur in $\epsilon$ in a recurrence assignment. In fact, such recurrence assignment is really very common and indispensible in practice.

Our algorithm solves the problem with the recurrence assignment with auxiliary variable VALUE as a temporary recorder for the expression value and the destination variable are eliminated from the symbolic predicate with procedure var_eliminate() before being assigned by procedure condition_effect () .

\section{Reduction Techniques}

There are two reduction techniques we used to battle the state-space explosion problem. Due to page-limit, we give a brief description of them.

\subsection{Inactive Local Variable Elimination}

The idea is that from some states, some variables will not be used until they are written again. Such variables are called inactive in such states and their values 
can be forgotten without affecting the behavior of the software implementation. Such a technique has been used heavily in tools like Spin 10], UPPAAL 4], SGM[16], and red 14 15]. But for systems with pointers, it is important to note that pointers used for indirect referencing are also implicitly read in the execution of the corresponding action. With this caution in mind, we develop a fixed-point procedure to derive an over-approximation local state predicate that describes the states in which a local variable is active. Once we find that a variable is inactive in all states described by a BDD, we can

- replace the values of those inactive local discrete variables in a state with zeros; and

- replace the values of those inactive local pointers in a state with nulls; With such replacements, we expect to greatly cut the complexity of our reachable state space representations.

However, it can be difficult to determine the exact description of a state set in which a local variable is inactive. In fact, we shall aim at constructing a local state predicate for an over-approximation of the active condition. Given a local discrete variable $x$, the local state predicate will be in $B\left(G^{d} \cup L^{d}-\{x\}, G^{p} \cup L^{p}\right)$. For a local pointer $x$, it will be in $B\left(G^{d} \cup L^{d}, G^{p} \cup L^{p}-\{x\}\right)$. That is, the over-approximation is described in terms of the variables, except $x[p]$, directly observable by the local process $p$. Then a lower approximation of the corresponding inactive condition of $x[p]$ is obtained by negating the just-obtained over-approximation of the active condition. By applying our technique to the MCS algorithm in figure 1, we find that

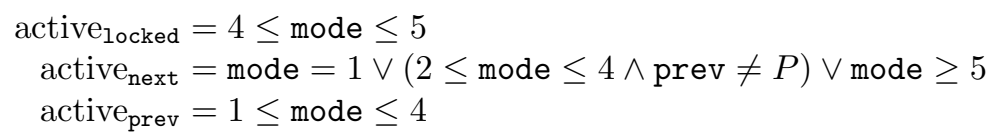

It shows that local variable locked, for example, will not be read and thus affect the system behaviors outside local modes 4 and 5 . The elimination of values of locked when it becomes inactive makes the state-space representation more concise and compact.

\subsection{Symmetry Reduction}

We follow the reduction framework in 8] to permute process identifiers to take advantage of the symmetry among processes running different copies of the same program. Our idea is to use the pointing-to relations of the global and local pointers to define a precedence relation among processes in a state, then permute the processes according to the precedence relation. We view the pointer datastructure as a directed graph. Each global pointer and each process is viewed as a node while the pointing-to relation is viewed as arcs from nodes to nodes. Thus the symmetry reduction for pointer data-structures has the flavor of graph isomorphism problem with node renaming, which is not yet known to be in PTIME. Intuitively, we want to keep as few data-structures, which are isomorphic, as possible. 


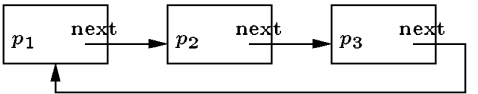

(a)

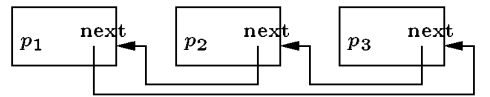

(b) after perumtation

Fig. 2. Anomaly of image false reachability

There are two challenges here. The first is how to design an efficient symmetry reduction strategy. For $m$ processes, we have $m$ ! different permutations and obvioously we do not want to try them all to find the best permutation. Our technique toward this challenge is to use binary permutations, which permute two processes each time, to compose full permutation. In theory, we know that all permutations can be constructed with a sequence of binary permutations. This is like to say that bubble-sort works for any sequence. However, binary permutations can create some data-structure configurations which are not reachable. For example, we may have $\mathcal{M}=3$, such that the local pointers next of the processes initially form the following static clockwise cycle in figure 2(a). If we choose to use the image cycle after binary permutation $\sigma=(132)$ as the representative, then the representative state in the equivalence class will be the counter-clockwise cycle shown in figure 2(b). But the problem is that the chosen counter-clockwise cycle image may never be reachable from the initial state if the cycle is a static one. We call this problem the anomaly of image false reachability. Although this is a possible cause for imprecision, we choose to live with it knowing that graph isomorphism problem can be too complex to solve.

With binary permutation, we have to design a predicate reverse $(i, j)$, for each $1 \leq i<j \leq m$ which characterizes those data-structure configurations in which processes $i$ and $j$ have to be permuted. This give us the second challenge, namely, how to design a criterion to determine when we need to permute two process identifiers. Our technique is to define an artificial distinct significance to each global and local pointer. For example, in the MCS algorithm, in our significance scale, the process pointed to by $L$ is much more significant than the others. Thus the process pointed to by $L$ should precede all other processes after the permutation. Basically, we assign the significance to global pointers according to their declaration order. The same is true among local pointers. Suppose local pointer next is declared before prev in MCS algorithm. Thus if neither of processes $i$ and $j$ are pointed to by $L$ and process $i$ 's local pointer next points to process $j$, then we know process $i$ cannot be preceded by process $j$ after the permutation. We have to consider the pointing-to relation of local pointer prev to decide the precedence between two processes only when we cannot decide their precedence with $L$ and next. In figure 3. we have drawn the four process network constructed by the MCS algorithm respectively before and after our permutation in figure 1 After the permutation, the network nodes are reordered in a linear sequence according to the queue formation. 

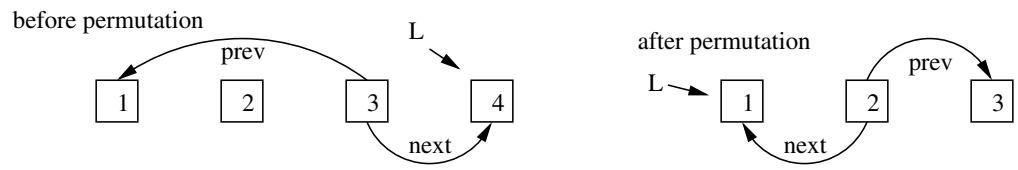

Fig. 3. Permutation of process identifiers

\section{Implementation and Experiments}

We implemented our techniques in a symbolic verification tool called red 14 15], which supports verification of timed automata 1 1 with a new BDD-like data structure for dense-time state-space representation. The reduction by inactive variable elimination is automatic. The symmetry reduction for pointer datastructure is invoked by option "Sp." We compared the performance of red, both with and without the symmetry reduction technique, with that of SMC 9 and Mur $\phi[11$ running in various options. Since neither SMC and Mur $\phi$ supports pointers, we have to use arrays in them to encode the pointers.

We have tested our implementation with three benchmarks. The first is the MCS locking algorithm whose red code can be found in our tool website. The safety condition to check is that no two processes are in the critical section at the same time. (Please check example 2.3.)

The second benchmark is a leader-election alogorithm. Each process has a local pointer parent. A set of symmetric processes randomly request to be a child of another process, who is not yet somebody's child. The process responds to a request will write its identifier to a global pointer respond_id. Then the requesting process will write the content of respond_id to its local variable parent. The processes use their variables parent to construct a dynamic forest structure. We want to make sure that at any time, at least some process is not somebody's child.

The third benchmark is a dynamic double-link cycle insertion and deletion algorithm. We have a set of symmetric processes which insert itself in and delete itself from the cycle maintained by two local pointers: next and prev. We also have a global pointer L pointing to the tail of the cycle. If there is no process in the cycle, then $\mathrm{L}=$ NULL. The safety condition to check is that when a process thinks it itself is in the cycle, global pointer L $\neq$ NULL.

The performance data table is in table 1 .

The first row of columns 3 to 11 are for the numbers of processes in the implementation. All the data is collected on a Pentium III $800 \mathrm{MHz}$ running Linux with 256MB. All data of red are collected with forward analysis (option -f). In each entry of the rows, the CPU times and memory consumptions (in kilobytes) are shown. The memory complexity for red is collected only for BDDs and their management.

The three benchmarks represent three different types of dynamic datastructures: doubly-linked queues, doubly-linked cycles, and forests with arbitrary 
Symmetric Symbolic Safety-Analysis of Concurrent Software

Table 1. Performance data table of three benchmarks

\begin{tabular}{|c|c|c|c|c|c|c|c|c|c|}
\hline \multirow{2}{*}{$\begin{array}{l}\text { benchmarks } \\
\text { MCS }\end{array}$} & Tools & Options? & 3 & 4 & 5 & 6 & 7 & 8 & 9 \\
\hline & \multirow[t]{4}{*}{ red } & \multirow[t]{2}{*}{$-\mathrm{fSp}$} & $0.98 \mathrm{~s}$ & $5.25 \mathrm{~s}$ & \begin{tabular}{c|c|}
$27.09 \mathrm{~s}$ \\
\end{tabular} & $141.56 \mathrm{~s} 9$ & $228.26 \mathrm{~s} 8$ & $\overline{8584.44 \mathrm{~s} 1}$ & $\overline{\overline{15072.26 \mathrm{~s}}}$ \\
\hline & & & $48 \mathrm{k}$ & $131 \mathrm{k}$ & $397 \mathrm{k}$ & $1064 \mathrm{k}$ & $3299 \mathrm{k}$ & $22824 \mathrm{k}$ & $197599 \mathrm{k}$ \\
\hline & & \multirow[t]{2}{*}{$-f$} & $2.33 \mathrm{~s}$ & $23.13 \mathrm{~s}$ & \multirow{2}{*}{\multicolumn{2}{|c|}{$\begin{array}{l}291.21 \mathrm{~s} 5442.33 \mathrm{~s} \\
12776 \mathrm{k} 234497 \mathrm{k}\end{array}$}} & \multirow{2}{*}{\multicolumn{3}{|c|}{ Not available }} \\
\hline & & & $139 \mathrm{k}$ & $1125 \mathrm{k}$ & & & & & \\
\hline & \multirow[t]{13}{*}{ SMC } & $-\mathrm{s} 1$ & \begin{tabular}{|r|}
$145.0 \mathrm{~s}$ \\
$\mathrm{C} / \mathrm{D}$
\end{tabular} & \multicolumn{6}{|c|}{ Not available } \\
\hline & & $-\mathrm{s} 2$ & $596.4 \mathrm{~s}$ & $>17 \mathrm{~h}$ & \multirow{2}{*}{\multicolumn{5}{|c|}{ Not available }} \\
\hline & & & $13472 \mathrm{k}$ & $\mathrm{N} / \mathrm{F}$ & & & & & \\
\hline & & \multirow[t]{2}{*}{$-\mathrm{s} 3$} & $600.3 \mathrm{~s}$ & $>17 \mathrm{~h}$ & \multirow{2}{*}{\multicolumn{5}{|c|}{ Not available }} \\
\hline & & & $13477 \mathrm{k}$ & $\mathrm{N} / \mathrm{F}$ & & & & & \\
\hline & & \multirow[t]{2}{*}{$-\mathrm{s} 4$} & \multicolumn{2}{|c|}{$1601.8 \mathrm{~s} 20252.6 \mathrm{~s}$} & \multirow{2}{*}{\multicolumn{5}{|c|}{ Not available }} \\
\hline & & & $13460 \mathrm{k}$ & $\mathrm{C} / \mathrm{D}$ & & & & & \\
\hline & & \multirow[t]{2}{*}{$-\mathrm{s} 5$} & $1624.0 \mathrm{~s}$ & $>17 \mathrm{~h}$ & \multirow{2}{*}{\multicolumn{5}{|c|}{ Not available }} \\
\hline & & & $13457 \mathrm{k}$ & $\mathrm{N} / \mathrm{F}$ & & & & & \\
\hline & & $-\mathrm{s} 6$ & $1600.3 \mathrm{~s}$ & $>17 \mathrm{~h}$ & & & t availa & able & \\
\hline & & & $13459 \mathrm{k}$ & $\mathrm{N} / \mathrm{F}$ & & & & & \\
\hline & & $-\mathrm{s} 7$ & $1620.8 \mathrm{~s}$ & $>17 \mathrm{~h}$ & & & t availa & able & \\
\hline & & & $13457 \mathrm{k}$ & $\mathrm{N} / \mathrm{F}$ & & & & & \\
\hline & Murphi & -sym1 & $0.18 \mathrm{~s}$ & $3.89 \mathrm{~s} 3$ & $771.83 \mathrm{~s}$ & $\mathrm{~T} / \mathrm{M}$ & & Not availal & ble \\
\hline & & -sym2 & $0.19 \mathrm{~s}$ & $3.54 \mathrm{~s} 1$ & $179.72 \mathrm{~s}$ & $\mathrm{~T} / \mathrm{M}$ & & Not availal & ble \\
\hline & & -sym3 & $0.18 \mathrm{~s}$ & $3.27 \mathrm{~s} 1$ & $142.47 \mathrm{~s}$ & $\mathrm{~T} / \mathrm{M}$ & & Not availal & ble \\
\hline & & -sym4 & $0.18 \mathrm{~s}$ & $3.29 \mathrm{~s} 1$ & $148.04 \mathrm{~s}$ & $\mathrm{~T} / \mathrm{M}$ & & Not availal & ble \\
\hline leader & red & -fSp & $0.02 \mathrm{~s}$ & $0.05 \mathrm{~s}$ & $0.10 \mathrm{~s}$ & $0.16 \mathrm{~s}$ & $0.25 \mathrm{~s}$ & $0.35 \mathrm{~s}$ & $0.54 \mathrm{~s}$ \\
\hline election & & & $17 \mathrm{k}$ & $38 \mathrm{k}$ & $69 \mathrm{k}$ & $113 \mathrm{k}$ & $171 \mathrm{k}$ & $246 \mathrm{k}$ & $337 \mathrm{k}$ \\
\hline & & $-\mathrm{f}$ & $0.02 \mathrm{~s}$ & $0.05 \mathrm{~s}$ & $0.10 \mathrm{~s}$ & $0.16 \mathrm{~s}$ & $0.25 \mathrm{~s}$ & $0.35 \mathrm{~s}$ & $0.54 \mathrm{~s}$ \\
\hline & & & $17 \mathrm{k}$ & $38 \mathrm{k}$ & $69 \mathrm{k}$ & $113 \mathrm{k}$ & $171 \mathrm{k}$ & $246 \mathrm{k}$ & $337 \mathrm{k}$ \\
\hline & SMC & $-\mathrm{s} 1$ & $0.3 \mathrm{~s}$ & $0.5 \mathrm{~s}$ & $0.3 \mathrm{~s}$ & $0.7 \mathrm{~s}$ & $4.8 \mathrm{~s}$ & $62.7 \mathrm{~s}$ & $2096.4 \mathrm{~s}$ \\
\hline & & & $1 \mathrm{k}$ & $7 \mathrm{k}$ & $34 \mathrm{k}$ & $193 \mathrm{k}$ & $1224 \mathrm{k}$ & $8444 \mathrm{k}$ & $68240 \mathrm{k}$ \\
\hline & & $-\mathrm{s} 2$ & $0.2 \mathrm{~s}$ & $0.2 \mathrm{~s}$ & $0.4 \mathrm{~s}$ & $2.4 \mathrm{~s}$ & $42.7 \mathrm{~s}$ & $1097.2 \mathrm{~s}$ & $34511.2 \mathrm{~s}$ \\
\hline & & & $1 \mathrm{k}$ & $7 \mathrm{k}$ & $29 \mathrm{k}$ & $135 \mathrm{k}$ & 681 & $3707 \mathrm{k}$ & $21799 \mathrm{k}$ \\
\hline & & $-\mathrm{s} 3$ & $0.2 \mathrm{~s}$ & $0.2 \mathrm{~s}$ & $0.4 \mathrm{~s}$ & $2.3 \mathrm{~s}$ & $29.5 \mathrm{~s}$ & $944.1 \mathrm{~s}$ & $16335.1 \mathrm{~s}$ \\
\hline & & & $1 \mathrm{k}$ & $7 \mathrm{k}$ & $28 \mathrm{k}$ & $134 \mathrm{k}$ & 567 & $3451 \mathrm{k}$ & $14964 \mathrm{k}$ \\
\hline & & $-\mathrm{s} 4$ & $0.2 \mathrm{~s}$ & $0.4 \mathrm{~s}$ & $0.4 \mathrm{~s}$ & $1.4 \mathrm{~s}$ & $9.2 \mathrm{~s}$ & $73.7 \mathrm{~s}$ & $619.0 \mathrm{~s}$ \\
\hline & & & $1 \mathrm{k}$ & $6 \mathrm{k}$ & $19 \mathrm{k}$ & $62 \mathrm{k}$ & 196 & $604 \mathrm{k}$ & 1857 \\
\hline & & $-\mathrm{s} 5$ & $0.3 \mathrm{~s}$ & $0.3 \mathrm{~s}$ & $0.4 \mathrm{~s}$ & $1.3 \mathrm{~s}$ & $8.9 \mathrm{~s}$ & $70.4 \mathrm{~s}$ & $591.0 \mathrm{~s}$ \\
\hline & & & $1 \mathrm{k}$ & $6 \mathrm{k}$ & $19 \mathrm{k}$ & $62 \mathrm{k}$ & 195 & $602 \mathrm{k}$ & 1851 \\
\hline & & $-\mathrm{s} 6$ & $0.3 \mathrm{~s}$ & $0.3 \mathrm{~s}$ & $0.5 \mathrm{~s}$ & $1.4 \mathrm{~s}$ & $9.1 \mathrm{~s}$ & $73.3 \mathrm{~s}$ & $621.0 \mathrm{~s}$ \\
\hline & & & $1 \mathrm{k}$ & $6 \mathrm{k}$ & $19 \mathrm{k}$ & $62 \mathrm{k}$ & 196 & $604 \mathrm{k}$ & 1857 \\
\hline & & $-\mathrm{s} 7$ & $0.2 \mathrm{~s}$ & $0.3 \mathrm{~s}$ & $0.4 \mathrm{~s}$ & $1.3 \mathrm{~s}$ & $8.8 \mathrm{~s}$ & $70.7 \mathrm{~s}$ & $592.6 \mathrm{~s}$ \\
\hline & & & $1 \mathrm{k}$ & $6 \mathrm{k}$ & $19 \mathrm{k}$ & $62 \mathrm{k}$ & 195 & $602 \mathrm{k}$ & 1851 \\
\hline & Murphi & -sym1 & $0.1 \mathrm{~s}$ & $0.1 \mathrm{~s}$ & $0.2 \mathrm{~s}$ & $2.81 \mathrm{~s}$ & $\mathrm{~T} / \mathrm{M}$ & Not av & ailable \\
\hline & & -sym2 & $0.1 \mathrm{~s}$ & $0.11 \mathrm{~s}$ & $0.18 \mathrm{~s}$ & $2.56 \mathrm{~s}$ & $\mathrm{~T} / \mathrm{M}$ & Not av & ailable \\
\hline & & -sym3 & $0.1 \mathrm{~s}$ & $0.12 \mathrm{~s}$ & $0.19 \mathrm{~s}$ & $2.57 \mathrm{~s}$ & $\mathrm{~T} / \mathrm{M}$ & Not av & ailable \\
\hline & & -sym4 & $0.1 \mathrm{~s}$ & $0.1 \mathrm{~s}$ & $0.2 \mathrm{~s}$ & $2.58 \mathrm{~s}$ & $\mathrm{~T} / \mathrm{M}$ & Not av & ailable \\
\hline double cycle & ared & -fSp & $0.06 \mathrm{~s}$ & $0.26 \mathrm{~s}$ & $0.95 \mathrm{~s}$ & $3.59 \mathrm{~s}$ & $14.86 \mathrm{~s}$ & $59.65 \mathrm{~s}$ & $228.72 \mathrm{~s}$ \\
\hline insertion & & & $16 \mathrm{k}$ & $38 \mathrm{k}$ & $98 \mathrm{k}$ & $254 \mathrm{k}$ & $618 \mathrm{k}$ & $1462 \mathrm{k}$ & $3418 \mathrm{k}$ \\
\hline & & $-\mathrm{f}$ & $0.93 \mathrm{~s}$ & $67.89 \mathrm{~s}$ & $>15 \mathrm{~m}$ & & Not a & available & \\
\hline & & & $165 \mathrm{k}$ & $10073 \mathrm{k}$ & $\mathrm{N} / \mathrm{F}$ & & & & \\
\hline & SMC & & & & No termi & ination & & & \\
\hline & Murphi & -sym1 & $0.1 \mathrm{~s}$ & $0.1 \mathrm{~s}$ & $0.11 \mathrm{~s}$ & $0.29 \mathrm{~s}$ & $2.13 \mathrm{~s}$ & $24.36 \mathrm{~s}$ & $290.26 \mathrm{~s}$ \\
\hline & & -sym2 & $0.1 \mathrm{~s}$ & $0.1 \mathrm{~s}$ & $0.11 \mathrm{~s}$ & $0.16 \mathrm{~s}$ & $0.53 \mathrm{~s}$ & $5.99 \mathrm{~s}$ & $66.59 \mathrm{~s}$ \\
\hline & & -sym3 & $0.1 \mathrm{~s}$ & $0.1 \mathrm{~s}$ & $0.11 \mathrm{~s}$ & $0.11 \mathrm{~s}$ & $0.12 \mathrm{~s}$ & $0.15 \mathrm{~s}$ & $0.17 \mathrm{~s}$ \\
\hline & & -sym4 & $0.1 \mathrm{~s}$ & $0.1 \mathrm{~s}$ & $0.1 \mathrm{~s}$ & $0.11 \mathrm{~s}$ & 0.1 & 0.13 & 0.15 \\
\hline
\end{tabular}

$\mathrm{s}(\mathrm{m}, \mathrm{h})$ : CPU time in seconds (minunts, hours); k: Memory in kilobytes;

C/D: core dumped; T/M: internal error, too many states; N/F: not finished; I/E: internal error 
number of children of each internal nodes. According to the data, only Mur $\phi$ outperforms red in one benchmark, the double-cycle insertions which generates the simplest data-structure configurations in all three benchmarks.

For the leader-election benchmarks, we found that our symbolic techniques is so powerful that the safety property is verified in our quotient-structure BDD without ever having to invoking the symmetry reduction. But for the other two benchmarks, symmetry becomes indispensible.

\section{References}

[1] R. Alur, C. Courcoubetis, D.L. Dill. Model Checking in Dense Real-Time, Information and Computation 104, pp.2-34 (1993).

[2] J.R. Burch, E.M. Clarke, K.L. McMillan, D.L.Dill, L.J. Hwang. Symbolic Model Checking: $10^{20}$ States and Beyond, IEEE LICS, 1990.

[3] M. Bozga, C. Daws. O. Maler. Kronos: A model-checking tool for real-time systems. 10th CAV, June/July 1998, LNCS 1427, Springer-Verlag.

[4] J. Bengtsson, K. Larsen, F. Larsson, P. Pettersson, Wang Yi. UPPAAL - a Tool Suite for Automatic Verification of Real-Time Systems. Hybrid Control System Symposium, 1996, LNCS, Springer-Verlag.

[5] R.E. Bryant. Graph-based Algorithms for Boolean Function Manipulation, IEEE Trans. Comput., C-35(8), 1986.

[6] E. Clarke, R. Enders, T. Filkorn, S. Jha. Exploiting symmetry in temporal logic model checking. Formal Methods in System Design 9, 77-104, 1996.

[7] D.L. Dill. The Murphi Verification System. CAV 1996, LNCS, Springer-Verlag.

[8] E.A. Emerson, A.P. Sistla. Utilizing Symmetry when Model-Checking under Fairness Assumptions: An Automata-Theoretic Approach. ACM TOPLAS, Vol. 19, Nr. 4, July 1997, pp. 617-638.

[9] A.P. Sistla, V. Gyuris, E.A. Emerson. SMC: A Symmetry-based Model Checker for Verification of Safety and Liveness Properties. TOSEM 9(2): Pages 133-166

[10] G.J. Holzmann. The Spin Model Checker, IEEE Trans. on Software Engineering, Vol. 23, No. 5, May 1997, pp. 279-295.

[11] C.N. Ip, D.L. Dill. Better Verification through Symmetry. FMSD 9(1/2):41-75, 1996.

[12] J.M. Mellor-Crummey, M.L. Scott. "Algorithms for Scalable Synchronization on Shared-Memory Multiprocessors." ACM Transactions on Computer Systems, Vol. 9, No.1, Feb. 1991, pp.21-65.

[13] K. Schmidt. How to claculate symmetries of Petri nets. Acta Informatica 36, 545-590, 2000.

[14] F. Wang. Efficient Data-Structure for Fully Symbolic Verification of Real-Time Software Systems. TACAS'2000, LNCS 1785, Springer-Verlag.

[15] F. Wang. Symbolic Verification of Complex Real-Time Systems with ClockRestriction Diagram, IFIP FORTE, August 2001, Cheju Island, Korea.

[16] F. Wang, P.-A. Hsiung. Efficient and User-Friendly Verification. IEEE Transactions on Computers, Jan. 2002, Vol. 51, Nr.1, ISSN 0018-9340, pp. 61-83. Preliminary materials of this paper also appears in proceedings of IEEE HASE'98, RTCSA'98, and IFIP FORTE'99. 